\title{
Electro-Chemical Degradation of Thin Film X2 Safety Capacitors
}

\author{
P L Lewin ${ }^{1}, \mathrm{~J}$ C Fothergill ${ }^{2}$ and S J Dodd ${ }^{3}$ \\ ${ }^{1}$ The Tony Davies High Voltage Laboratory, University of Southampton, UK \\ ${ }^{2}$ City University London, London, UK \\ ${ }^{3}$ Department of Engineering, University of Leicester, UK
}

\begin{abstract}
There is some field evidence that certain manufactured batches of thin film X2 capacitors are more susceptible to electro-chemical corrosion than others. Studies undertaken at the University of Leicester, City University London and the University of Southampton have investigated this degradation mechanism, developed underlying theory for this behaviour and validated the theory using data from damp heat testing. This paper details the anatomy of thin film X2 capacitors, details the principal mechanisms of degradation and breakdown before explaining the electrochemical corrosion mechanism and associated loss of capacitance. The effects of this degradation mechanism on other properties of the capacitor are shown to be minimal as evidenced by dielectric spectroscopy and other measurements. The ultimate conclusion is that unlike other types of capacitor, a pre-defined drop in initial capacitance does not signify end of useful life and for specific applications end of life of an X2 capacitor should be defined as the minimum value of X2 capacitance that will ensure reliable operation of a given circuit.
\end{abstract}

\section{Keywords-thin film capacitors, electrochemical degradation}

\section{INTRODUCTION}

Thin film safety capacitors are defined as capacitors that meet the requirements of IEC60384-14. This standard incorporates 7 groups of tests that cover standard capacitor properties: resistance to heat, vibration, shock and solvents, damp heat performance, impulse voltage endurance, charging and discharging properties, RF characteristics and passive/active flammability tests. For capacitors designed not to be connected directly to earth, the standard qualifies a capacitor series as $\mathrm{X} 1, \mathrm{X} 2$ or $\mathrm{X} 3$ where the number refers to the peak voltage that the capacitor can safely handle $(4 \mathrm{kV}, 2.5$ $\mathrm{kV}$ and $1.2 \mathrm{kV}$ respectively). A key design feature is that $\mathrm{X}$ capacitors will always fail-safe, i.e. never fail as a short circuit but lose capacitance until they are equivalent to an open circuit. X1 and X2 capacitors find wide application in mains powered equipment and are commonly used for EMI suppression where their role is to essentially protect electronic circuitry from overvoltage transients that can occur on a mains supply.

Thin film capacitors use a dielectric that is typically 6 to $8 \mu \mathrm{m}$ thick. Polyester and more recently polypropylene are typically used by safety capacitor manufacturers. The dielectric film has a metallised layer that is around 10 to $14 \mathrm{~nm}$ thick and this layer is usually aluminium or zinc or a mixture of the two. A typical capacitor for use at mains voltages will consist of a roll of interleaved films of $10 \mathrm{~mm}$ to $20 \mathrm{~mm}$ in width, where the width and length of the roll will determine the overall capacitance (lengths of up to $5 \mathrm{~m}$ are not uncommon). The films in the roll are interleaved so that alternate electrode layers are flush with one of the flat sides of the roll while on the other side there is a margin of a $\sim 1 \mathrm{~mm}$ of dielectric only. The roll is pressed to flatten it and a Schooping [1] applied (basically a zinc spray) on the original flat sides to provide electrical continuity for the alternate electrodes that are flush with that side. The Schoopings are connected to pins which are a standard pitch apart and the capacitor potted in a plastic box using an epoxy resin.

\section{A. 'Clearing' - a mechanism for loss of capacitance}

The design of the capacitor facilitates a 'self-healing' mechanism, often referred to as clearing. A well-known and reported failure of dielectric film is breakdown due to the presence of a high electric field which locally exceeds the intrinsic breakdown strength of the material. Inevitably when this occurs the film is punctured and a short circuit results. Failure may be due to local imperfections in the dielectric, inclusion of foreign particles (conducting or insulating) or failure may be due to external events causing a transient high field across the film e.g. lightning or switching impulses impressed on the applied voltage. However, due to their design, thin film capacitors do not fail short circuit. If there is breakdown, then dissipation of the energy resulting from the local short circuit is sufficient to evaporate the electrodes in the vicinity of the breakdown site (see Figure 1). The removal of the electrodes associated with a 'clearing' event has a marginal effect on the overall capacitance reducing it by less than $0.01 \%$. At the point of production all $\mathrm{X} 1$ and $\mathrm{X} 2$ capacitors are tested to ensure that they are free from defects that might lead to excessive clearing.

There is growing field evidence that some safety capacitors, used in mains equipment, are exhibiting a graceful degradation process, where over a period of months/years there is a loss of capacitance until the capacitor is purely a very high impedance. Work completed at the Universities of 
Leicester, Southampton and City University London has investigated the causes of this degradation process and has identified this process is loss of electrode area caused by electrochemical corrosion. Analysis of damp heat test data supports this hypothesis. Following an explanation of the degradation process itself, damp heat results are presented and analysed, measurement of key capacitor properties of degraded capacitors presented before discussing the implications of the findings and outlining the conclusions resulting from this study.

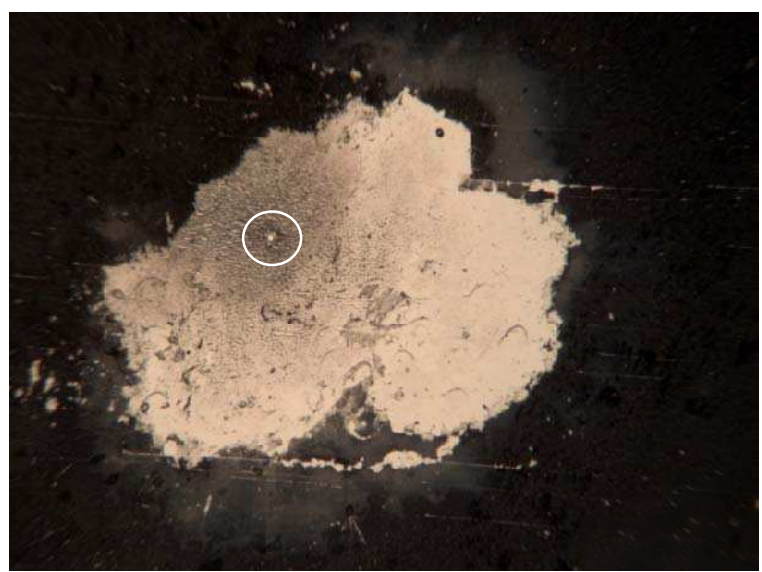

Fig. 1. Typical clearing found in a X2 capacitor: The puncture site is circled and the loss of electrode material around the site is extensive.

\section{ELECTROCHEMICAL CORROSION}

This is not a new or unknown mechanism; work by Taylor in 1984 [2] theoretically calculated the voltage required for electrochemical corrosion to occur in a high voltage capacitor as being in excess of $250 \mathrm{~V}$. In a 1994 review of capacitor ageing mechanisms, Reed and Cichanowski include electrochemical corrosion as a known problem [3]. However, this may have been more recently 'forgotten' by thin film capacitor manufacturers and changes in terms of materials and manufacturing processes may have unwittingly created an increased likelihood of susceptibility to this failure mechanism in some production batches of $\mathrm{X}$ capacitors.

\section{A. A graceful; degradation mechanism}

For electrochemical corrosion to occur within a safety capacitor certain conditions need to be satisfied: first, there must be a sufficiently high ac voltage present and second there must be water molecules in very close proximity to the electrode. Unpublished experiments conducted at the University of Leicester have revealed that the rate of corrosion is significantly increased at elevated temperatures in a high humidity environment. Electrochemical corrosion does not occur if there is no applied ac voltage or if the applied voltage is dc or the capacitor is in a dry environment. The electrochemical reaction takes place relatively rapidly once all conditions are met and results in the formation of a metal oxide that has very good insulating properties. The oxide is slightly thicker than the original metalized layer (where the increase in thickness is of the order of 1 or 2 nanometres). Consequently the change in capacitor dimension due to electrochemical corrosion will be less than a micron in any direction and will not lead to mechanical failure of the component. If considering the standard capacitor model then the series resistance of a capacitor suffering electrochemical corrosion will increase slightly meaning that under full load the internal temperature of the capacitor may be higher by a few degrees but not high enough to cause thermal failure. On the other hand, the insulation resistance will also increase as a consequence of electrochemical corrosion, inferring that dc leakage current will be reduced. However the process of electrochemical corrosion does not significantly affect the dielectric, particularly at the frequency of $50 \mathrm{~Hz}$, and this is evidenced by measurements of capacitance and $\tan \delta$ of degraded capacitors, examples of which are shown in Figure 2.

Forensic analysis of degraded capacitors has revealed that the electrode is corroded from the margin rather than the thicker edge that is electrically bonded to the Schooping. In other words water molecules are passing through the Schooping and move across the small insulation gap to react with the edges of the electrodes that are connected to the Schooping on the other side of the capacitor. Consequently, the dielectric properties of the capacitor (apart from the obvious loss of capacitance) are unaffected.

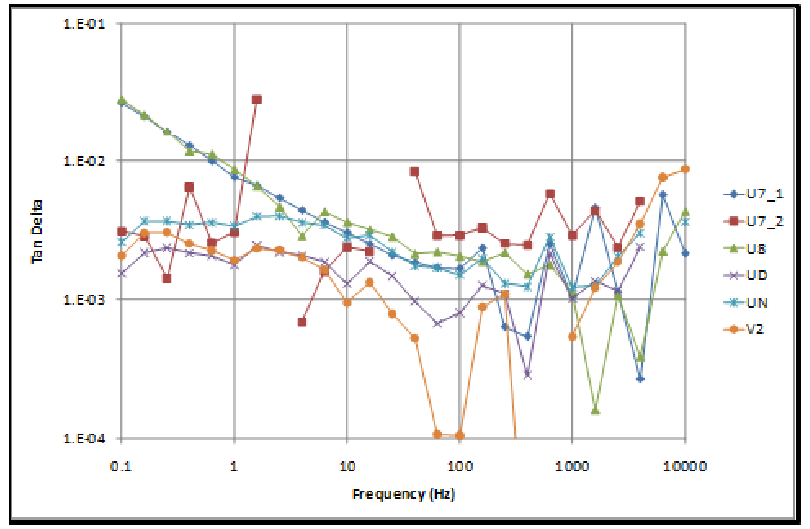

(a)

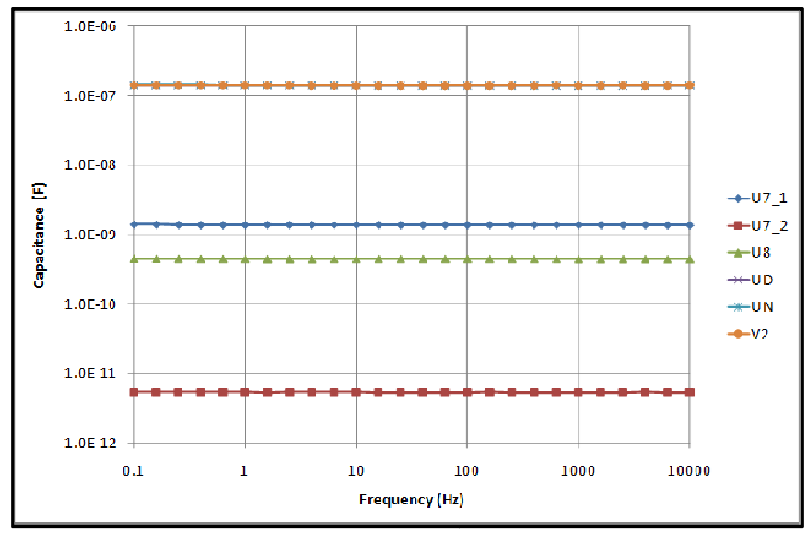

(b)

Fig. 2. (a) Capacitance as a function of frequency for degraded capacitors of original nominal capacitance of $150 \mathrm{nF} \pm 20 \%$. (b) Tan delta measurements for the same set of capacitors. The increase in tan delta is not significant even in the case of severly degraded capacitors (U8, U7_1 and U7 2). (Considerable noise is present in the tan delta measurements less than $\sim 10^{-\overline{3}}$.) 
The dielectric measurements show no reason why the maximum withstand voltage of the capacitor should decrease after electrochemical corrosion. Under normal ambient conditions it may take several years before the loss of capacitance due to electrochemical corrosion is observed. Since other properties of the capacitor are largely unaffected, this process is a "graceful" degradation mechanism, where the rate determining step is hypothesized as the time taken for water molecules to diffuse into the capacitor epoxy, pass through the Schooping and diffuse along the margin in order to react with the electrode edge.

\section{The RATE DETERMINING STEP}

An individual capacitor's susceptibility to electrochemical corrosion can be determined using an accelerated lifetime test. A typical test would apply rated or operational ac voltage to a capacitor in a high temperature high humidity environment (e.g. $85^{\circ} \mathrm{C}$ and $85 \%$ relative humidity). Relative performance is evaluated by measurement of the capacitance at regular intervals (for example, every 100 hours over a test duration of 1000 hours). To date, there is no agreed international standard test for assessing susceptibility to electrochemical corrosion of thin film capacitors. Typical results for 30 capacitors from a production batch that was known to have susceptibility to electrochemical corrosion are shown in Fig. 3.

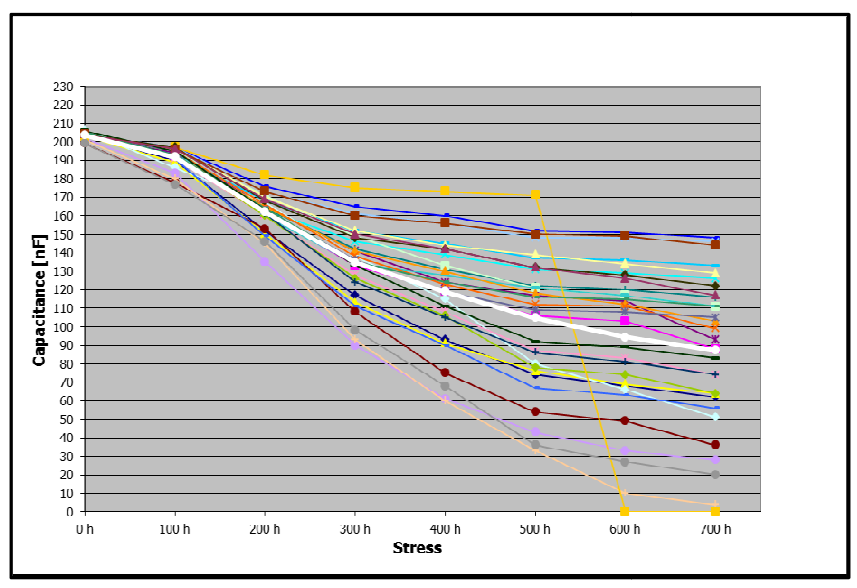

Fig. 3. Measurement of capacitance as a function of time for 30 off $220 \mathrm{nF}$ $\mathrm{X} 2$ capacitors taken from a production batch known to have susceptibility to electrochemical corrosion. Test conditions; 230 Vac applied, $85{ }^{\circ} \mathrm{C}$ and $85 \%$ RH for 700 hours. Note one capacitor failed open circuit between 500 hours and 600 hours, this data was not used in subsequent analysis.

With reference to Fig. 3, there are some common features in the data set. For the majority of the capacitors under test there is little change in capacitance over the first hundred hours, this is then followed by a rapid change over the next three to four hundred hours and then a slower rate of change over the last two hundred hours. An explanation of this behaviour could be that it takes an initial period of time for water to reach the edge of an electrode (i.e. time required for water to diffuse from the external environment through the epoxy, across the Schooping and along the margin). Once water is present there is a loss of capacitance due to electrochemical corrosion but as more of the electrode is replaced with non-conducting oxide the time taken for water to diffuse to the edge of the electrode increases. If the rate determining step is due to diffusion then the loss of capacitance after the ingress time taken for water to reach the electrode edge should be controlled by Fick's Law. Fick's law states that the time to diffuse is proportional to the square of the distance over which the diffusion takes place.

With reference to Fig.3, for each capacitor the damp heat data is a series of $\mathrm{N}$ capacitance measurements taken at fixed time intervals under constant ambient conditions. If there are $\mathrm{K}$ capacitors being monitored, the data set can be defined as;

$$
\left\{\left(t_{j}, c_{k}\right), j=0, \ldots, N, k=0, \ldots K\right\}
$$

It is proposed that a good model to fit to the damp heat test data for the capacitance, $c$, of the kth capacitor that has an initial capacitance measurement of $C_{0}$, would be;

$$
\mathrm{c}\left(t, t_{i}, m_{k}, n_{k}\right)=\left\{\begin{array}{c}
C_{0}, 0<t \leq t_{i} \\
C_{0}-m_{k}\left(t-t_{i}\right)^{n_{k}}, t>t_{i}
\end{array}\right.
$$

Where $t_{i}$ is the ingress time, $m_{k}$ and $n_{k}$ are the capacitor specific scaling coefficient and exponent respectively. The exponent effectively describes the 'shape' of the capacitance loss. If the loss of capacitance is determined by a diffusion mechanism then it would be expected that values of $n_{k}$ would be less than one and most likely between 0.3 and 0.7 .

\section{A. Determining the exponent.}

For each capacitor it is necessary to determine estimates of $t_{i}, m_{k}, n_{k}$ (i.e. $\left.\hat{t}_{i}, \widehat{m_{k}}, \widehat{n_{k}}\right)$ such that the error between the model and measurements over the duration of the experiment are minimised. Let the error of the kth capacitor, $\varepsilon_{k}$, be defined as

$$
\varepsilon_{k}=\sum_{j=1}^{N} c\left(t_{j}, \widehat{t_{l}}, \widehat{m_{k}}, \widehat{n_{k}}\right)-c_{j}
$$

Where $c_{j}$ is the measured capacitance at the jth time step. The task is then to find the best estimates of $t_{i}, m_{k}, n_{k}$ such that for the kth capacitor this error is minimised, i.e.

$$
\arg \min _{t_{i}, m_{k}, n_{k}} \varepsilon_{k}
$$

It is assumed that measurement error in the experimental data is normally distributed. This analysis has been used on the measurement data shown in Fig 3 (ignoring the one capacitor that failed open circuit during the experiment). Figure 4 details a typical result.

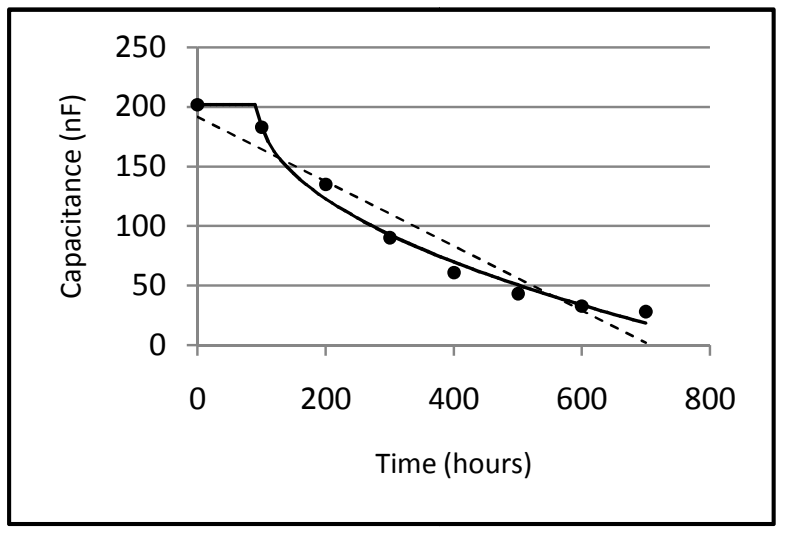

Fig. 4. Comparison of damp heat measurement data (black circles) and the best fit curve of Equation 2 for one of the $220 \mathrm{nF}$ capacitors detailed in Fig 3. The dashed line represents the best fit straight line approximation. 
With reference to Fig. 4 there is good agreement between the best fit curve and the experimental values, especially when compared to the result obtained for a best-fit straight line approximation. Analysis of estimated of $t_{i}$ and $n$ for $88220 \mathrm{nF}$ capacitors that have been damp heat tested are shown in Fig.5

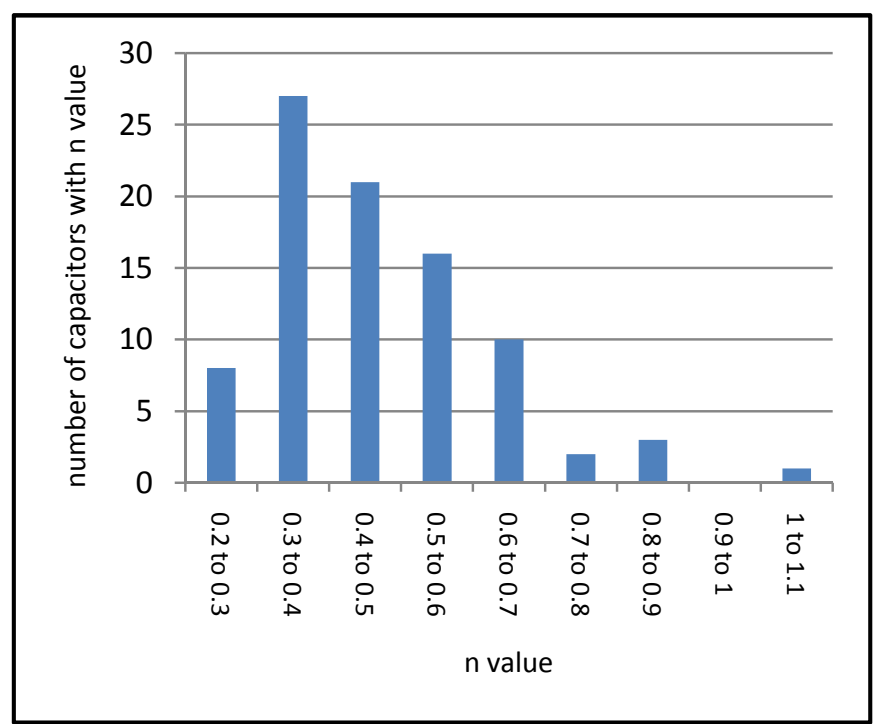

(a)

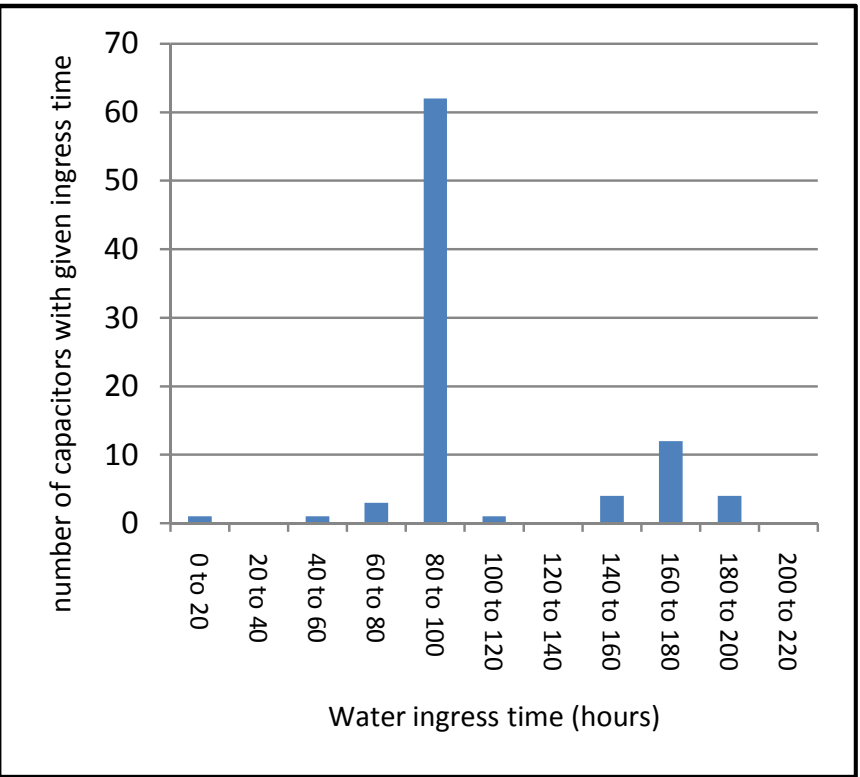

(b)

Fig. 5. Histograms showing distribution of best fit parameters for $88220 \mathrm{nF}$ capacitors that have been damp heat tested. (a) the range of exponent values, (b) the range of ingress times.

With reference to Fig.5, for the 88 capacitors that have been analysed $86 \%$ have a model where the best fit value of exponent lies in the range $0.3<\mathrm{n}<0.7$ and $70 \%$ have an estimate of water ingress time between 80 and 100 hours. Although, the data set is small, this is compelling evidence that under conditions of high temperature and humidity, following water ingress, the rate of capacitance loss is governed by the diffusion of water molecules to the retreating electrode edge.

\section{DISCUSSION}

Analysis of $\mathrm{X}$ capacitors that have lost capacitance due to electrochemical corrosion reveals that the dielectric is unaffected. Other types of capacitor can become unsafe if their properties, e.g. dissipation factor in the case of electrolytic capacitors, change significantly from nominal values. As a result of this certain types of capacitor will have defined end of life criteria which are expressly defined in their data sheets. Often end of life is defined in terms of percentage loss of capacitance from initial value, however, this does not hold for metallised film capacitors. In the case of X safety capacitors, end of life will be application dependent, for example if used for EMI suppression significant capacitance (e.g. more than $50 \%$ ) could be lost before performance was compromised and other components exposed to potentially harmful transient voltages.

The use of damp heat testing allows assessment of likely susceptibility to electrochemical corrosion for production batches of $\mathrm{X}$ capacitors and it is reasonable to set acceptance criteria for such tests (e.g. to be acceptable, all capacitors tested must not lose more than a certain percentage of their initial value over the duration of the test). Analysis of damp heat test data reveals that the process of electrochemical corrosion is largely governed by water ingress and diffusion to the reaction site. Designs of $\mathrm{X}$ capacitor that are resistant to corrosion can be readily assessed using damp heat testing. Resistance to corrosion can be achieved by various means, for example; internal series connected capacitors have the advantage that the voltage across the electrodes is significantly reduced; or changing the materials used for the electrode or dielectric or to pot the capacitor may increase corrosion resistance.

\section{CONCLUSIONS}

Thin film safety capacitors used in harsh environments may lose capacitance over time if they are susceptible to electrochemical corrosion. This is a graceful degradation process such that it may be several years before the loss of capacitance becomes significant. The corrosion process itself is governed by diffusion and this theory has been validated by analysis of damp heat data for capacitors from production batches known to be susceptible. In practical applications, end of useful life of an X Capacitor should be defined based on the minimum value of capacitance that will still ensure reliable operation of a given circuit.

\section{REFERENCES}

[1] M.U. Schoop: Verfahren zum Herstellen dichter, metallischer Überzüge, Patent-Nr. 258505, DE, 1909.

[2] D.F. Taylor, "On the Mechanism of Aluminum Corrosion in Metallized Film AC Capacitors" IEEE Trans. Elec.Insul. Vol. EI-19(4), 288-293 (1984)

[3] C.W. Reed and S.W. Cichanowski "The Fundamentals of Aging in HV Polymer-film Capacitors", IEEE Transactions on Dielectrics and Electrical Insulation, 1 (5), 904 - 922 (1994) 